\title{
The Coupling Effect of Tourism Industry and New Urbanization in Guanzhong Region of Shaanxi Province under the Background of Global Tourism
}

\author{
Qihong Tan
}

Leisure Management school of Xi’an Eurasia University, Xi'an, Shaanxi, 710065

Keywords: coupling effect; new urbanization; Guanzhong Area

\begin{abstract}
In the new historical period, new urbanization has emerged as a dilemma in response to the international economic sluggishness, longstanding domestic structural contradictions, the gradual disappearance of the "demographic dividend", and the cyclical deceleration of the economy, which has become a socio-economic structure. The key to the transformation of all-round transformation and economic growth driven by exports and investment is the transformation of domestic demand. In this context, exploring the relationship between tourism industry and new urbanization and promoting the coordinated development of tourism industry and new urbanization has become an important topic of common concern for all walks of life. It not only has an important guiding role in the sustainable development of tourism industry, also is of great practical significance to the coordinated development of urban and rural areas and the scientific process of new urbanization.
\end{abstract}

\section{Introduction}

Urbanization of tourism, from the earliest foreign scholar Patrick Malins, has gradually attracted the attention and attention of scholars at home and abroad. In the research on the impact of tourism urbanization, foreign scholar Mullins combined the two tourist cities on the Gold Coast and Sunshine Coast in Australia to analyze the impact of tourism urbanization on the local area; Allen et al pointed out that tourism urbanization is a natural environment for coastal destinations, Different levels of influence in the social environment, ecological environment, etc.; the domestic research on "urbanization of tourism" began in 1999. Lu Lin and other scholars have studied the impact of tourism urbanization on local economy, environment, society and culture from different perspectives. The role was discussed. In short, the studies on the impact of domestic and foreign scholars on tourism urbanization mostly focus on the qualitative research on the connotation, characteristics and effects of tourism urbanization, and on the coupling and coordination between tourism industry and urbanization. Research needs to be further studied.

\section{Mechanism of Coordinated Development of T-U-E Systems}

By referring to previous studies, coupling can be defined as a phenomenon in which two or more systems (or elements) interact with each other and influence each other. Coupling degree is used to analyze the degree of interaction between systems (or elements). The degree of coupling coordination is used to measure the extent to which the internal and external elements of the system are consistent with each other in the development process. The tourism industry, the new urbanization and the ecological environment system are an organic whole that influences, interacts with and promotes each other. The tourism industry promotes urban and rural development through the development of rural tourism products, promotes the development of many industries in the town through the industrial linkage effect, relieves employment pressure in the urban and rural areas through employment effects, and strengthens the urban foreign exchange through high degree of openness to promote the process of new urbanization. The new urbanization construction supports the development of the tourism industry by providing support for the development of the tourist industry, such as customer markets, basic services, technical talents, and tourism space and tourism image carriers. The ecological environment is the basic condition for the existence and 
development of the expansion of the tourism industry and new urbanization, which directly restricts the development level of the tourism industry and new urbanization. Only when tourism industry development, new urbanization expansion and ecological environment protection are in harmony, can a virtuous cycle be formed to achieve sustainable development. Therefore, the mechanism of the three coupled development can be summarized as follows: Under the basic conditions of the ecological environment, the tourism industry and the new urbanization system promote and interact with each other and jointly affect the development of the ecological environment. Among them, the tourism industry is to promote the engine, the new urbanization is to support the protection, the ecological environment is the basic conditions.

Let $\mathrm{Vi}$ be the actual index value of the order parameter evaluation index $\mathrm{Ai}(\mathrm{i}=1,2, \ldots, \mathrm{n})$. In order to convert the value of the evaluation index Ai into an orderly efficiency coefficient to the system, construct the power function $\mathrm{D}(\mathrm{Ai})$ as:

$$
D\left(A_{i}\right)=\left\{\begin{array}{lrr}
\left(V_{i}-a_{i}\right) /\left(\beta_{i}-a_{i}\right), A_{i} \text { is } & \text { Positive } & \text { effect } \\
\left(\beta_{i}-V_{i}\right) /\left(\beta_{i}-a_{i}\right), A_{i} i s & \text { negtive } & \text { effect }
\end{array}\right.
$$

Among them, $\alpha \mathrm{i}$ and $\beta \mathrm{i}$ are the upper and lower limit values within the actual value range of the system evaluation index $\mathrm{Ai}$, and can be estimated by referring to the standard value, the base period value, and the like. Positive efficacy indicators indicate that the higher the indicator value, the greater the positive impact on the system. Conversely, the negative efficacy indicator indicates that the higher the indicator value, the greater the negative impact on the system. Because the tourism industry system and the new urbanization system are very complex and large systems, comprehensive measurement of them needs to take into account all the indicators involved in the system, to reflect the comprehensive results of the role of indicators, but also to reflect the various systems. Overall coupling coordination degree [6]. To this end, the comprehensive utility evaluation function of the tourism industry system and the new urbanization system is:

$$
f(x)=\sum_{i}^{m} a_{i} D(A), g(Y)=\sum_{i}^{m} b_{i} D(A)
$$

Among them, $\mathrm{f}(\mathrm{X})$ and $\mathrm{g}(\mathrm{Y})$ are the comprehensive utility evaluation functions of tourism industry system and new urbanization system respectively; ai and bi are respectively assigned weights of each index, which can be determined by entropy weighting method. Coupling degree is a measure of the degree of strength and weakness of the influence and interaction between the order parameters. Using the coupling coefficient evaluation model in physics, the coupling model of tourism industry and new urbanization is constructed:

$$
E=\frac{\sqrt{f(x) f(y)}}{f(x)+f(y)}
$$

The value of the coupling degree $\mathrm{E}$ is bounded by $(0,1)$. When $\mathrm{E}=1$, it means that the system is in a completely correlated state, and the coupling degree is the highest. When $\mathrm{E}=0$, it means that the internal and external elements of the system are completely independent, and the coupling degree is the lowest. According to the value of the coupling degree, the coupling process can be divided into four stages: low-level coupling, running-in and high-level coupling. The corresponding coupling degree ranges in each stage are $(0,0.3],(0.3,0.5],(0.5,0.8],(0.8,1)$. In these four stages, the tourism industry and the new urbanization construction have experienced a weak coupling effect from the initial two, and a certain degree of coupling effect has occurred. However, the phenomenon of mutual inhibition exists to a certain extent, and then evolves into a benign coupling between the two, which promotes to a certain extent, and finally forms a highly coupled relationship of mutual promotion and coordinated development. 


\section{Visitors' Region Tourism Industry and New Urbanization Research}

Using 2005-2016 as the evaluation year, the degree of coupling coordination between tourism and new urbanization was studied, and the following conclusions were obtained: (1) After evaluating the tourism index and the new urbanization index of five prefecture-level cities It is learnt that every city is in the phase of simultaneous development of tourism and new urbanization. (2) Through the analysis of the coupling and coordination degree between tourism and new urbanization, it was found that the coupling of tourism and new urbanization in five cities was in an increase phase. The degree of coupling between Xian City and Xianyang City increased rapidly; The Baoji City and Weinan City was a medium level coupling stage.Only Tongchuan City was a low level coupling stage.

Therefore, this article proposes the following suggestions on how to realize the coordinated development of tourism and new urbanization in Guanzhong region: First, to perfect the top-level design of the coupling and coordinated development of tourism and new urbanization. At present, tourism and new urbanization are highly valued. The tourism industry and new urbanization in the Guanzhong region must achieve rapid development. However, at present, there is a lack of coordination between the development of tourism in the region and the process of new-type urbanization, and it is in a state of self-government. Therefore, it is necessary to emphasize the improvement of the top-level design, and on the basis of a comprehensive assessment of the tourism resources and new urbanization conditions in various regions, emphasis is placed on the coordinated and coordinated development of the tourism industry in the Guanzhong region and the new type of urbanization. Each city must choose according to local conditions according to its own development conditions. The right coupling development mode of coordination. The second is to strengthen the institutional guarantee for the coupling and coordinated development of tourism and new urbanization. In order to promote the coordinated and coordinated development of the tourism industry in the Guanzhong region and the new type of urbanization, we must ensure the following aspects. First, we must promote the transformation of government functions, strengthen the government's macro-control and coordination functions, weaken the direct intervention in the tourism market, and give full play to the decisive role of the market in the allocation of tourism resources. Second, we must adopt market-based capital operation methods to promote investment entities. Diversification, making full use of foreign capital, corporate funds, and private capital to jointly develop tourism and promote revenue sharing. Third, we must strengthen environmental protection and governance. We must adhere to scientific development and reasonable development in the process of tourism development and new urbanization. Pay attention to improving tourism service level. The third is to choose the mode of coordinative development of tourism and new urbanization in line with local conditions. As mentioned earlier, the development of the tourism industry in the Guanzhong region is different from the new urbanization stage. The appropriate development model should be selected according to the different conditions of each city. Xian City, which has rich tourism resources and a relatively new basis for urbanization, should strengthen tourism promotion and marketing, increase city awareness, and attract domestic and foreign tourists to drive the further development and promotion of local tourism, and it must promote timely. The transformation and upgrading of tourism products, on the other hand, also need to improve the infrastructure construction of local transportation, hotels, etc., and improve the regional tourism reception capacity and accessibility. Xianyang City and Baoji City and Weinan City, which is rich in tourism resources and has a good basis for new urbanization, should step up publicity and marketing efforts for tourism scenic spots in order to increase the popularity of scenic spots to attract more tourists, but also pay attention to the construction of tourism supporting service facilities. For Tongchuan, where the relative lack of tourism resources and the poor basis of new urbanization, should seize the opportunities of western development, precision poverty alleviation, and the rejuvenation of old Shaanxi-Shaanxi revolutionary regions, optimize the spatial distribution of cities and towns, and accelerate the development of new urbanization. In particular, the construction of supporting facilities in cities and towns will increase the accommodation space and development level of towns and cities and provide a good basis and conditions for the development 
of tourism.

\section{Conclusion}

This paper constructs a coupling evaluation model and evaluation index system of tourism industry and new-type urbanization, and combines the actual situation in Guanzhong area to empirically analyze the coupling effects of local tourism industry and new urbanization. The main conclusions of the study include: First, there are obvious coupled development characteristics between the tourism industry system and the new urbanization system; secondly, the tourism industry and the new urbanization level in the Guanzhong region have increased substantially during the period from 2005 to 2016; Thirdly, the coupling degree between tourism industry and new urbanization in the Guanzhong region has always been rampant. Fourth, the coupling degree of tourism and urbanization in five cities in Guanzhong is different. Among them, the coupling degree of Xi'an is the highest, and the coupling degree of Tongchuan is the lowest.

\section{Acknowledgment}

Shaanxi Provincial Department of education, subject number (17JK1032)

\section{References}

[1] Fang Yelin, Huang Zhenfang, Duan Zhongxian, et al. Coupling and Coordinating Research on China's Tourism Development and Ecological Environment [J]. Economic Geography, 2013, 33(12) : 195.

[2] LU Lin. Tourism Urbanization: An Important Topic of Tourism Research[J]. Tourism Journal, 2005, 20(4).

[3] Huang Zhenfang, Wu Jiang, Hou Guolin. Preliminary Study on Urbanization of Tourism: A Case Study of the Metropolitan Continuum in the Yangtze River Delta [J]. Resources and Environment in the Yangtze Basin, 2010,9(2).

[4] Li Peng. The model of tourism urbanization and its regulation research [J]. Social scientists, 2004, (4).

[5] Zhang Hongchao, Zhao Lijie. Evaluation of the Coupling Effects of Industrial Clusters and Regional Economy [J]. Statistics and Decision, 2013, (5) 